\title{
Smart Metro - Rail System
}

\author{
Srivas M C, Suman S Hosmane, Kumarnarayan Bhat, Dilip Kumar M S, Shreenivas B
}

Department of Telecommunication Engineering, BMS College of Engineering, Bengaluru, India

\begin{abstract}
Railway transport system has proved to be a very sturdy and convenient mode of transport over the centuries. It still serves as the economic and most efficient means of mass transport in many countries.

It is a widespread practice even today that most operations are manually carried out, leading to several dangerous accidents and mismanagement of the system. When it comes to the matter of scores of lives, error margin is of utmost importance to ensure an efficient and safe mode of travel. There is an utter need for a system that provides automation of the critical systems that play a pivotal role in the smooth functioning.

The focus remains on certain key functions including locomotion, data logging for position, speed and health of he locomotive. This can increase the safety levels, while also reducing the time for relief in-case of unfortunate emergencies.
\end{abstract}

Keywords - Accidents, Automation, Emergencies, Mass Transport, Safety Levels.

\section{INTRODUCTION}

There have been instances many tragedies in the rail industry, even in developed countries due to the negligence of the man power involved in the operations. According to a census conducted in 2016, it was estimated that about $87 \%$ of the train accidents occurred in India was due to human error. Moreover, $2 \%$ accidents are due to failure of equipment, $2 \%$ are caused due to sabotage; $6 \%$ accidents are due to incidental factors. The short comings in the present system are overcome using various sensor networks interfaced with an embedded micro-controller. The sensors constantly update the micro-controller with the updated value almost continuously. The controller is programmed to take appropriate action based on the inputs from the sensor network. Working principle remains quite simple and cost effective.

In response to developing autonomous systems that can ease the operation of metro rail service and limiting manual interference a few ideas have been discussed in the recent past. This research paper develops a particular type of automated metro rail system designed to have mainly two modes of operation. Er. Thakurendra Singh et.al [1] proposes a train model which is equipped with a controller that enables the automatic stopping of the train from station to station. Thabit Sultan Mohammed et.al [2] discusses about a process of a prototype for a driverless train implemented using a PIC microcontroller. V.Sridhar [3] describes the working using a microcontroller with the RF receiver and the voice recorder chip with speaker. The whole system is attached to the vehicle (BUS or Train). The encoded RFID tags are placed in the BUS stops or the railway stations. H. Douglas et al [4] described a paper about maintaining service quality and managing increased demand with improving energy efficiency. Factors like driving style, sensitivity analysis above and underground are assessed to bring out safety and energy saving solutions in the metro system.

\section{SYSTEM ARCHITECTURE}

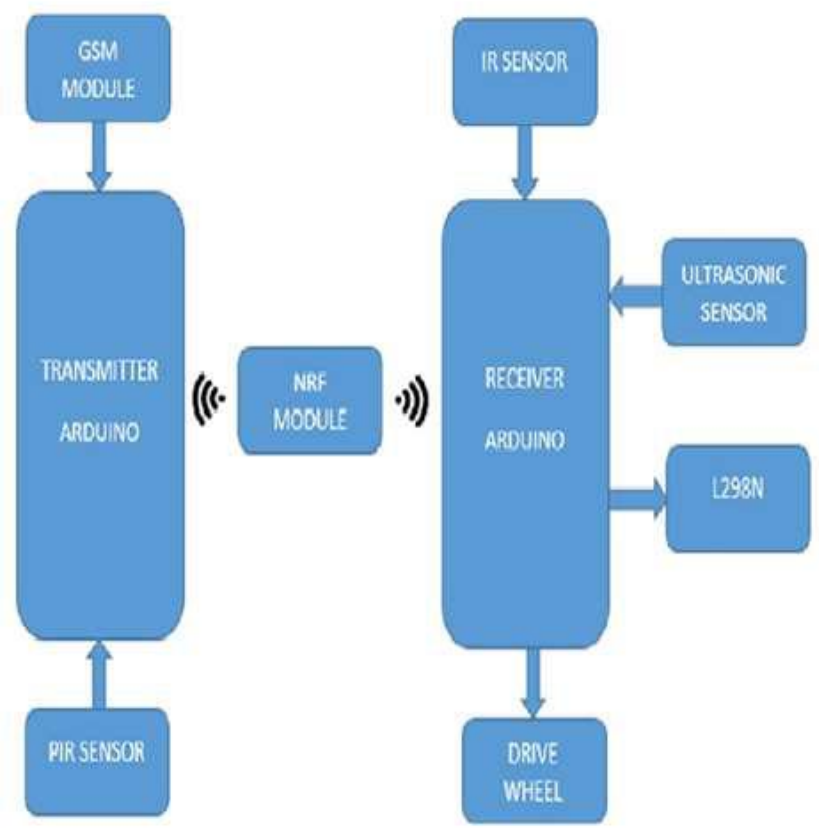

Fig.1: Basic system architecture

There are two sections that are necessary for the working of the complete system. The transmitter section which depicts the train station uses the Arduino to obtain inputs from the near range frequency module and the PIR sensor then send required messages to the receiver section. The receiver section which comprises of the operation of the train uses Arduino along with near range frequency module, IR sensors, DC motors and power supplies to run the train model. Near range frequency module at the receiver end receives certain commands from the transmitter station to perform the major operations of the 
whole system. IR sensors detect the path along which the train moves with the help of the DC motors. A GSM model is integrated at the train station to provide the authorities and customers with the latest information of the train.

\section{SYSTEM DESIGN AND METHODOLOGY}

\section{A. Control Site}

All the instructions are passed on to the locomotive from this location. Automated Control or Manual Control commands are transmitted by the controller through the wireless modes. The following components comprise of the control site.

1) Messaging Unit: To enhance the convenience of the passengers and the authorities, a SIM900 module which is a Quad-band GSM/GPRS compatible with cell phone is integrated to provide automated updates about the train's arrival and departure.

2) Train Arrival Detector: A passive infrared sensor (PIR sensor) is integrated for the purpose of detecting an incoming locomotive. When the train arrives, it is made to stop by sending commands using near range frequency modules while the GSM operation is carried out simultaneously.

3) NRF Transmitter: Wireless Monitoring of the locomotive is achieved through an NRF24101 module which is a low power half-duplex wireless communicator.

4) Transmitter Arduino: This acts as a means of User Interface through the connected laptop. It makes use of the IDE and COM port in order to take in inputs from the user and at the same time provide information about the messages sent across. All the above listed components are directly interfaced, and the inputs are used differently based on the choice of user i.e. the automated or manual mode of operation brings into action different sets of interfaces.

\section{Working of Control Site}

- The various components on the controller are interfaced with the Arduino Microcontroller at the controller site.

- PIR Sensor comes to play for the automated actions. It senses the incoming train to mark the arrival and initiates the stopping and door operations.

- GSM Module send updates through SMS when either a train is set to leave or has just arrived. The signals from the PIR sensor and command from the control room sets off the GSM Module when the train is detected or when the train is about to leave.

\section{B. Locomotive}

1) NRF Receiver: NRF24101 module is integrated to coordinate with the IR sensors and motors to carry out the normal working of the train.

2) Obstruction Detection: This system comprises of an ultrasonic detector. The sensor works on the principle of SONAR. The ultrasonic sensor uses sonar to determine distance to or from an object. It detects any obstruction approaching the train from the opposite direction and automatically stops to avoid any mishap.

3) L298N: Every train is necessarily designed with a door to facilitate the entry and exit of the passengers. This prototype is equipped with an automated door, with both 'CLOSE' and 'OPEN' operations. This bidirectional movement is achieved with a single drive motor

4) Door IR Sensor: For the safety purpose it is enabled with an IR Sensor which can detect and incoming person, during the closing operation so that it does not harm any individual and can be retracted in such situations.

5) Receiver Arduino: It acts as a hub for all the components at the receiver end. However unlike at the transmitter site, there is no visual interface for displaying out at various stages.

\section{Working of Locomotive}

All the components present on the locomotive are interconnected through the Arduino microcontroller.

- Commands sent from the wireless medium from the controller are received via the Receiver NRF Module and further processed by the Arduino. Various conditions are possible and each condition has been programmed to initiate particular action in the prototype.

- Microcontroller receives input from the Ultrasonic sensor and based on the value received, further action are either carried out or the entire system is halted.

- L298N H-bridge comes to play when the station arrives. The door can be opened and closed with variable timing.

- The IR Sensor placed at the door gives continuous reading to actuate the motion of the door. This feature helps in preventing casualties to passengers while entering and exiting.

\section{IMPLEMENTATION METHODS}

Both the methods use wireless communication in their working.

Fig. 2 illustrates the manual mode in detail. Here, the operator can exercise control over each operation of the locomotive. When the operator chooses to start the train, 
the train receives the command wirelessly to starts it operation from the station and the customer also receives a message. Now the locomotive runs along the track and checks for any obstruction in front. If any, it will automatically stop at a safe distance. When the operator chooses to stop the train, a stop command is received at the locomotive end to stop the train and perform the next action if necessary.

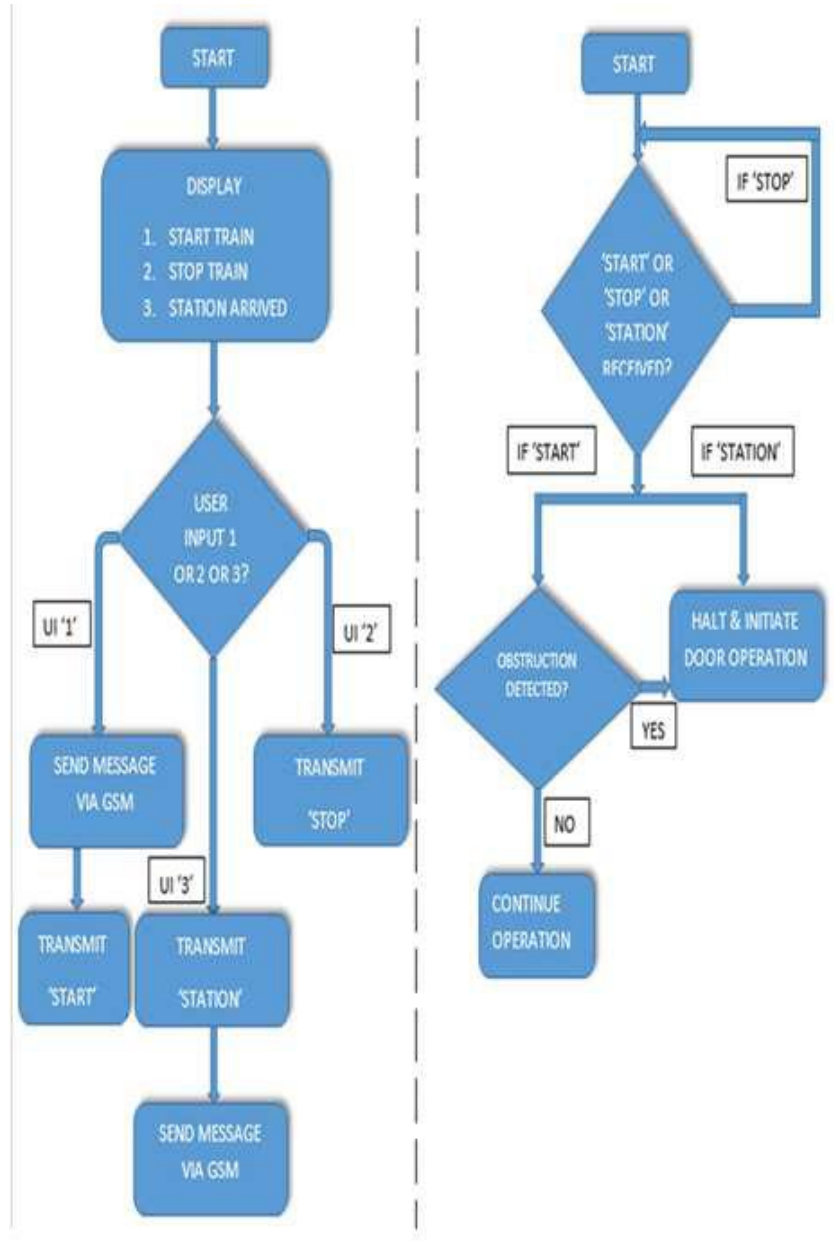

Fig.2. Flowchart of Manual Operation

Fig. 3 illustrates the step by step process of automated mode.

At the transmitter end, the 'Start' command is sent wirelessly and the locomotive will verify to start its operation. While the locomotive is in motion it checks for any obstruction and if there is any, it will automatically stop on its own.

When the transmitter station detects the arrival of the train, it sends a station command wirelessly to stop the locomotive so that the passengers can exit or enter at the station.

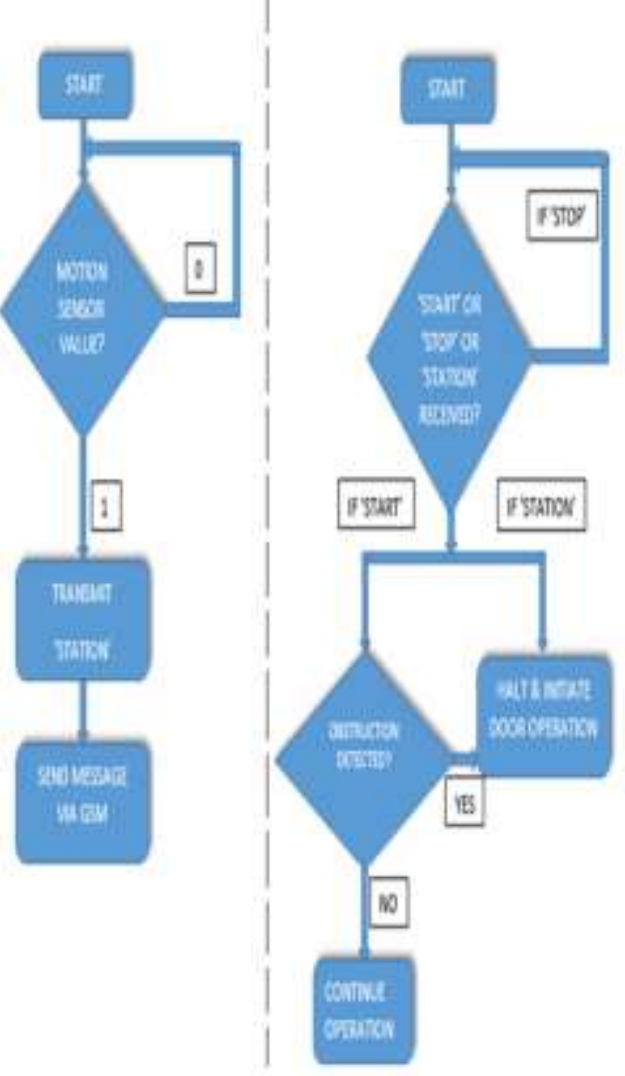

Fig.3. Flowchart of Automated Operation

The hardware components that were used in the working of the system broadly consist of the following:

i) Control station

ii) Prototype

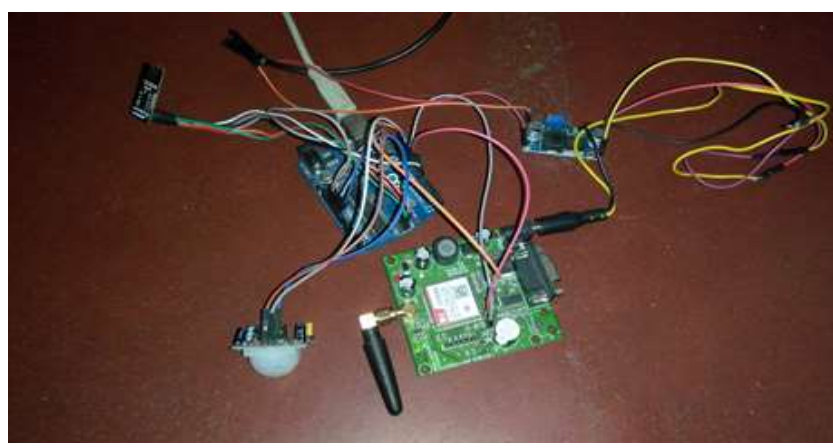

Fig.4. Control Station

Fig. 4 shows the control station consists of arduino, PIR sensor for detecting the arrival of train at the station, NRF module to wirelessly communicate with the metro train and a GSM module to send messages to the customers about the train arrival and departure. 


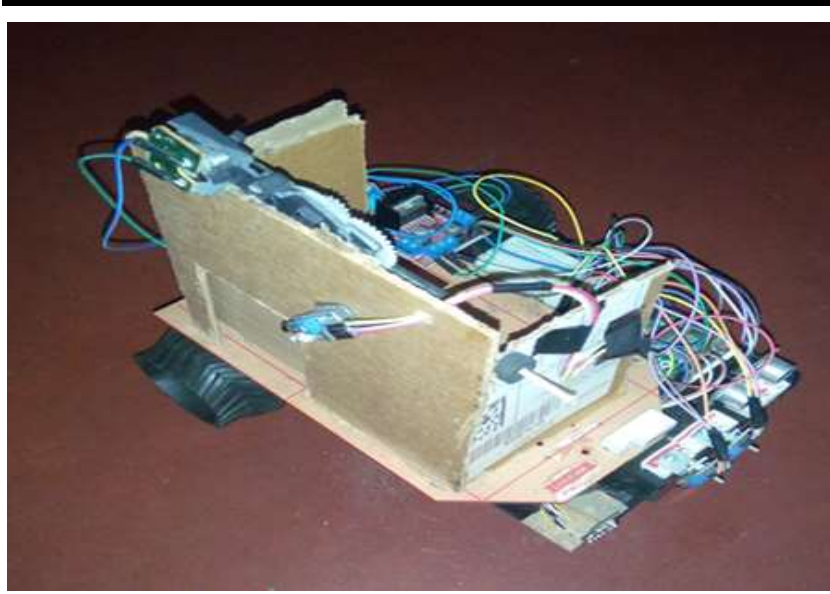

Fig.5: Prototype

Fig. 5 shows the prototype that was implemented - Smart metro, which mainly uses a microcontroller, NRF modules and motors to exhibit the driverless metro train operation which runs on a defined path. It also has NRF module on the left for wireless communication with the control station. Ultrasound sensors detect any obstruction in front of it, so that the metro train would stop and prevent any accident within a specified range. The door operation in the prototype is done by using a motor to open and close the door. Instructions are sent by the arduino as programmed. The IR sensor helps in detecting any obstruction during closing of door to prevent any risk for the passengers getting on or off the train in real life scenario.

\section{RESULT}

The implementation of this system in two modes produced consistent results in all the trial runs of the metro train.

Fig. 6 shows the user interface at the control station and GSM operations. The operator at the control station has the control to start or stop the train at the station so that the passengers can exit and enter the train. The door operations are carried out automatically and is programmed within the prototype to perform opening and closing of door. The train is ready to be started once these operations are finished. Manual mode gives the operator more control and he can manage the system at each stage of operation. In contrast automatic mode uses the capability of the entire system to the fullest extent by working entirely on its own till the station arrives. GSM operations are also performed simultaneously.

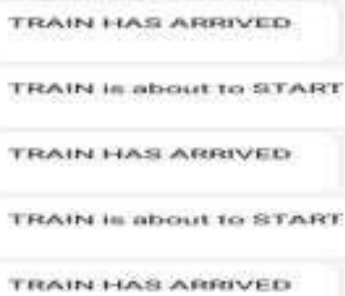

Fig.6: user interface at the control station and GSM operations.
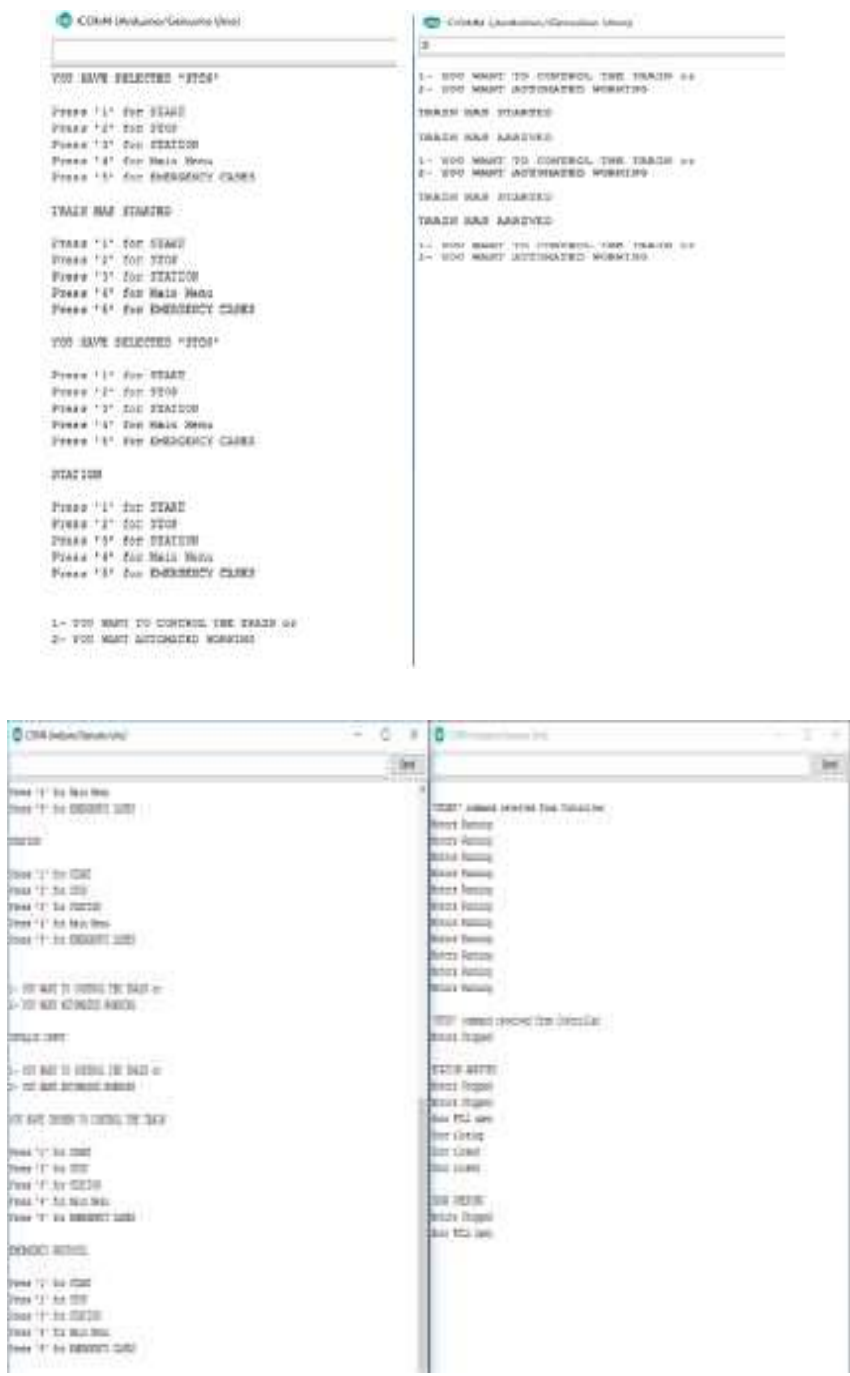

Fig.7: Emergency case operation

Fig 7 illustrates the emergency operation that is performed when the operator senses the danger in the prototype. The train is made to be stopped and the doors are open so that the passengers can exit safety if needed. A message is sent to the passengers' secondary number and emergency authorities are also notified by a message using GSM.

\section{CONCLUSION}

The autonomous capability of the metro train with 
multiple safety features and technical advancements have been implemented in this system. It has produced successful and consistent results in both methods using the available cost effective resources.

A simple prototype has been implemented with the idea of implementation in real life in Indian scenario which would help in easy and faster transportation of people. It would also reduce the number of traffic jams and air pollution to a very big extent. This would not only help the authorities and the customers to travel safely but also provide the entire population with cleaner environment.

High quality and durable sensors and devices with less maintenance can be added to make this system work in large scale areas and slowly replace existing manual systems. Having a data logging feature can help in the long run. Attaching various sensors in different areas of the locomotive can aid in collecting vital data which can be further processed to tune the working for the best fit curve to obtain best efficiency.

\section{ACKNOWLEDGMENT}

We would like to acknowledge the support and encouragement of the management of BMS College Engineering, Bengaluru. We also acknowledge our department and particularly, our guide, Shreenivas.B for providing all the resources, constant guidance and advice which helped us to bring our idea into a concrete form.

The project and paper would be a highly impossible task to achieve if it was not for their kind and patient guidance.

We thank Dr. Rajeshwari M Hegde, Head of the Department, Telecommunication Engineering, for encouraging and providing opportunity to carry out this project.

\section{REFERENCES}

[1] Auto Metro Train Shuttle between Two Stations Er. Thakurendra Singh, Nidhi Singh, Man Mohan Sharma, Rishabh Bhargav and Virendra Singh. International Journal of Electrical and Electronics Research ISSN 2348-6988 (online) Vol. 3, Issue 2, (274-276), April - June 2015.

[2] Full Automation in Driverless Trains: A Microcontroller-Based Prototype, Thabit Sultan Mohammed, Wisam Fahmi Al-Azzo, Mohammed Ahmed Akaak and Mohammed Laheeb Suroor. IJAREEIE, Vol. 3, Issue 7, July 2014, ISSN (Print) : 2320 - 3765, ISSN (Online): 2278 - 8875

[3] Automated system design for Metro train V.Sridhar, IJCSE Vol. 1 No.01 September 2012 ISSN : 23197323
[4] H. Douglas, C. Roberts, S. Hillmansen, "Optimising energy saving in metro systems through characteristic evaluation" IEEE, May 2016, Online ISBN: 978-178561-293-0

[5] B.W.C. Cooke. "Proposed New London Underground". The Railway Magazine (London) 101 (648): 279-281. April 1955.

[6] S. Cappaert-Blondelle. Metro Automation Facts, Figues and Trends. The International Association of Public Transport (UITP). Technical report. Belgium. 2012.

[7] J.M. Erbina, and C. Soulasa. Twenty Years of Experiences with Driverless Metros in France. VWT 19 proceedings. Dresden. 2003.

[8] Transportation system division. The Dubai Metro, the World's Longest Fully Automated Metro Network. Mitsubishi Heavy Industries

[9] A. Gonzales-Gil, R. Palacin, P. Batty and J. P. Powell, "A Systems Approach to Reduce Urban Rail Energy Consumption," Energy Conversion and Management, vol. 80, pp. 509-524, 2014. J. Wang, Fundamentals of erbium-doped fiber amplifiers arrays (Periodical styleSubmitted for publication), IEEE J. Quantum Electron., submitted for publication.

[10] UITP Europe, "Mandatory Exclusion of Urban Rail From the Scope of the Interoperability and Safety Directives Within the 4th Railway Package," UITP , Brussels, 2014.

[11] http://arduinobasics.blogspot.in/2014/06/433-mhz-rfmodule-with-arduino-tutorial.html 